\title{
Brown adipose tissue activity is reduced in women with polycystic ovary syndrome
}

\author{
Flávia R Oliveira ${ }^{1,2}$, Marcelo Mamede 3 , Mariana F Bizzi ${ }^{4}$, Ana Luiza L Rocha', Cláudia N Ferreira5, \\ Karina B Gomes ${ }^{6}$, Ana L Cândido ${ }^{4}$ and Fernando M Reis' \\ 1Department of Obstetrics and Gynecology, Universidade Federal de Minas Gerais, Belo Horizonte, Brazil, ${ }^{2}$ Odete \\ Valadares Maternity Hospital, Fundação Hospitalar do Estado de Minas Gerais, Belo Horizonte, Brazil, ${ }^{3}$ Department \\ of Anatomy and Imaging, ${ }^{4}$ Department of Internal Medicine, ${ }^{5}$ Technical College, and ${ }^{6}$ Department of Clinical and \\ Toxicological Analyses, Universidade Federal de Minas Gerais, Belo Horizonte, Brazil \\ Correspondence \\ should be addressed \\ to $\mathrm{F} M$ Reis \\ Email \\ fmreis@ufmg.br
}

\begin{abstract}
Objective: To evaluate whether brown adipose tissue (BAT) activity is altered in women with polycystic ovary syndrome (PCOS), and whether BAT activity correlates with plasma levels of irisin, a myokine that can induce BAT formation. Design: We performed a cross-sectional study including women with PCOS $(n=45)$ and a healthy control group $(n=25)$ matched by age and body mass index (BMI).

Methods: BAT activity was measured using ${ }^{18} \mathrm{~F}-\mathrm{FDG}$ positron emission tomography-computed tomography (PET-CT) and plasma irisin levels were measured by a validated enzyme immunoassay.

Results: Total BAT activity was significantly reduced in women with PCOS (maximal standardized uptake value (SUV max $_{\text {): }}$ median $7.4 \mathrm{~g} / \mathrm{mL}$, interquartile range 0.9-15.4) compared to controls (median $13.0 \mathrm{~g} / \mathrm{mL}$, interquartile range 4.7-18.4, $P=0.047$ ). However, this difference was no longer significant after adjustment for waist circumference, a surrogate marker of central adiposity. In the PCOS group, BAT activity correlated negatively with BMI (Spearman's $r=-0.630$, $P=0.000)$ and waist circumference $(r=-0.592, P=0.000)$ but not with plasma irisin levels.

Conclusions: BAT activity was reduced in women with PCOS possibly due to increased central adiposity. In PCOS women, BAT activity did not correlate with plasma irisin levels.
\end{abstract}

\section{Introduction}

Brown adipose tissue (BAT) has been long known in small mammals and newborns to maintain body temperature through thermogenesis (1). In contrast to the energystoring function of white adipose tissue, BAT is a highly energetic tissue with a unique mitochondrial expression of uncoupling protein-1 (UCP-1) that converts the cell respiration electrochemical gradient into heat, using glucose and fatty acids as fuels $(2,3,4)$.

Although some indirect evidence had led a few authors to postulate the presence of BAT in adult humans (5), it could be demonstrated only with the availability of positron emission tomography (PET) with ${ }^{18} \mathrm{~F}$-fluorodeoxyglucose $\left({ }^{18} \mathrm{~F}\right.$-FDG), a functional imaging method that evaluates areas of high metabolic activity
European Journal of Endocrinology

(2019) 181, 473-480
$(4,6,7,8)$. BAT cells are found in adult humans mainly in the supraclavicular, cervical and paraesternal regions (9). This discovery attracted great interest and led to the hypothesis that BAT distribution and function may be altered in metabolic diseases $(4,6,9,10)$.

Polycystic ovary syndrome (PCOS) is a common endocrine disorder that affects $5-15 \%$ of women of reproductive age. It is characterized by clinical and/or biochemical hyperandrogenism, ovulation failure and the presence of enlarged and/or polycystic ovaries in ultrasound images (11). Functional abnormalities of the adipose tissue have been reported in women with PCOS, primarily linked to insulin resistance, even in the absence of obesity (12). Yuan et al. demonstrated that BAT activity

Published by Bioscientifica Ltd. 
was dramatically decreased in a rat model of PCOS and that BAT transplantation reversed PCOS-like features such as estrous cycle irregularity and insulin resistance (13).

During the past decade, skeletal muscle has also been identified as an endocrine organ that releases cytokines and other peptides collectively named myokines. Irisin is an exercise-induced myokine that has been proposed to mediate the beneficial effects of physical exercise on metabolism $(14,15,16)$. The active irisin molecule derives from proteolytic cleavage of fibronectin type III domaincontaining 5 (FNDC5) and induces 'browning' of white adipocytes through the activation of UCP-1, leading to increased cell respiration and energy expenditure (15, $16,17,18)$. Irisin levels are positively associated with BMI and muscle mass (19). However, patients with type 2 diabetes have paradoxically lower irisin levels despite the association of this condition with obesity (17); therefore, the relationship between irisin and insulin resistance is complex and not fully understood $(17,20)$.

In women with PCOS, the evidence about irisin regulation remains largely conflicting $(21,22,23)$. A study found high serum irisin levels when hyperandrogenism was part of the PCOS phenotype (21), but a recent meta-analysis showed that, with adjustment for BMI, circulating irisin levels are similar in PCOS patients and healthy controls (22). In addition, BAT activity had not been previously assessed in women with PCOS, and we hypothesized that BAT activity and its relation with irisin might be a novel metabolic pathway disrupted in PCOS, with implications for the development of future treatments. Therefore, the aim of this study was to evaluate whether BAT activity is altered in women with PCOS and if it correlates with body measures or with plasma irisin levels.

\section{Subjects and methods}

\section{Study participants and ethical approval}

This was a cross-sectional study including 70 women aged 18-45 years divided into a group with PCOS $(n=45)$ and a healthy control group $(n=25)$. The groups were matched by age and BMI based on group means without individual pairing (24), and we stopped enrolment when both groups had reached the minimum sample size with similar age and BMI. Participants were enrolled from April 2015 to September 2017 at the outpatient facilities of two teaching hospitals in Belo Horizonte, Brazil. The study was approved by the Research Ethics Committee of Universidade Federal de Minas Gerais (Protocol ID 17127713.2.0000.5149) and all patients signed an informed consent.

The diagnosis of PCOS was made according to the 2003 Rotterdam ESHRE/ASRM PCOS Consensus Workshop Group diagnostic criteria (25). As per the Rotterdam criteria, we excluded patients with hypo or hyperthyroidism, hyperprolactinemia, 21-hydroxylase deficiency, Cushing's syndrome and androgen-producing tumors. To reduce potential confounders, we also excluded patients who had used antiandrogen drugs in the last six months or any hormones or metformin in the last 2 months. Finally, for safety concerns about the use of intravenous ${ }^{18} \mathrm{~F}-\mathrm{FDG}$, we excluded pregnant or puerperal women, women who were breastfeeding, and patients with diabetes or oral glucose intolerance. The control group consisted of women with regular menstrual cycles and without any clinical or biochemical hyperandrogenism or polycystic ovarian morphology and with no use of hormonal therapy or metformin in the past 2 months.

\section{Antropometric measures, lifestyle and laboratory assessment}

Body weight, height and waist circumference were measured by the same professional with the participants wearing lightweight clothing without shoes and BMI was calculated according to the standard formula (weight in kilograms divided by squared height in centimeters).

Physical activity was assessed through the International Physical Activity Questionnaire, quantified in min/week and classified into very active, active or sedentary as detailed elsewhere $(26,27)$. Women were classified as alcohol drinkers if they reported any quantity of alcohol consumption in the past month and as smokers if they smoked at least one cigarette per day $(28,29)$.

Blood samples were collected at 9:00 h after an overnight fast between the 3rd and 5th days of menstrual cycle. For serum collection, blood was withdrawn in a plain tube, allowed to clot and centrifuged for $10 \mathrm{~min}$ at $1500 \mathrm{~g}$ at room temperature. For plasma collection, blood was withdrawn in an EDTA-coated tube and centrifuged for $10 \mathrm{~min}$ at $2500 \mathrm{~g}$ at $4^{\circ} \mathrm{C}$. The serum and plasma aliquots were stored at $-80^{\circ} \mathrm{C}$ until assayed.

Serum levels of follicle-stimulating hormone (FSH), thyroid-stimulating hormone (TSH), prolactin, glucose, insulin, total cholesterol, high-density lipoprotein (HDL) cholesterol, low-density lipoprotein (LDL) cholesterol and triglycerides were measured using commercial assays (30). Total testosterone was assayed by a competitive 
immunoassay (Vitros® system, Ortho Clinical Diagnostics, Raritan, NJ, USA). Lipid accumulation product (LAP) index was calculated as abdominal circumference $(\mathrm{cm})$ $58 \times$ triglycerides (millimol/liter) (31).

\section{Plasma irisin assay}

Plasma irisin levels were assayed using a commercial ELISA kit (CUSABIO®, catalog number CSB-EQ027943HU), following strictly the manufacturer's instructions, in duplicate and with the operator blinded to the patient group. The intra-assay and inter-assay coefficients of variation, using three samples of known concentration, were $<8 \%$ and $<10 \%$, respectively. The assay sensitivity was $0.78 \mathrm{ng} / \mathrm{mL}$.

\section{Brown adipose tissue activity measurement}

BAT activity was measured using ${ }^{18}$ F-FDG PET-CT with cold activation in the morning after 9-12 $\mathrm{h}$ fasting. The method of cold activation has been previously described and validated (32). In brief, the participants remained in a room at $19^{\circ} \mathrm{C}$ for $2 \mathrm{~h}$ wearing only underwear and a thin, sleeveless short gown with an insulation effect of 0.18 clo (33), which corresponds to $18 \%$ of the insulating value of clothing needed to maintain a person in comfort sitting at rest in a room at $21^{\circ} \mathrm{C}$ with air movement of $0.1 \mathrm{~m} / \mathrm{s}$ and humidity less than 50\% (34). During the cold exposure no shivering activity was self-reported by the participants or noted by the researchers. After $1 \mathrm{~h}$ resting at $19^{\circ} \mathrm{C}$, ${ }^{18} \mathrm{~F}-\mathrm{FDG} \quad(0.1 \mathrm{mCi} / \mathrm{kg})$ was administered intravenously and the subjects remained in the same cold conditions for another hour. Afterward, a whole-body PET-CT scan was performed at $20^{\circ} \mathrm{C}$ using a dedicated PET-CT system (Discovery PET-CT 600, GE Healthcare). Low-dose CT without contrast enhancement was performed first and used for attenuation correction and anatomic image fusion $(90 \mathrm{kV}, 10-120 \mathrm{mAs}$ (smart $\mathrm{mA}$ ) with $3.75 \mathrm{~mm}$ section thickness). The PET images were reconstructed in a $192 \times 192$ matrix, using an Ordered Subsets Expectation Maximization (OSEM)-like algorithm, with 2 iterations and 24 subsets.

${ }^{18}$ F-FDG uptake in fat areas identified by CT was quantified using a dedicated workstation (Xeleris, GE Healthcare) as described elsewhere (35). BAT activity was expressed in $\mathrm{g} / \mathrm{mL}$ as the maximum standardized uptake value $\left(\mathrm{SUV}_{\max }\right)$, defined as the tissue radioactivity concentration $(\mathrm{KBq} / \mathrm{mL})$ divided by the injection dose per lean body mass $(\mathrm{KBq} / \mathrm{g})$. In each one of four body regions (cervico-thoracic, axillary, thoracic, and abdominal) a region of interest (ROI) was delimited around the most dense uptake zone and used to calculate the SUV $\mathrm{max}_{\text {. }}$ The highest BAT uptake value was used as the total BAT activity. For categorical analysis, the presence of active BAT was defined as a $\mathrm{SUV}_{\max }$ of at least $2.0 \mathrm{~g} / \mathrm{mL}$, as previously described (6). All images were evaluated by two independent readers (experienced nuclear physician and radiologist), blinded to the clinical findings and laboratorial results.

\section{Statistical analysis}

Sample size calculation indicated that 24 participants per group would be sufficient to detect a minimum difference of $5 \mathrm{~g} / \mathrm{mL}$ (0.8 standard deviation) in BAT activity with 95\% confidence and 80\% statistical power.

Continuous variables were tested for normal distribution by Shapiro-Wilk test. Normally distributed variables were expressed as means \pm standard deviation (s.D.) and compared between groups using unpaired Student's $t$ test, whereas variables that departed from normality were expressed as medians and quartiles and compared by the non-parametric Mann-Whitney $U$ test. Differences between groups were adjusted for potential confounders by analysis of covariance (ANCOVA) (36). Categorical variables were submitted to chi-square test with continuity (Yates) correction or Fisher's exact test, as appropriate. Linear correlations were evaluated by Spearman's rank correlation coefficient. We used Prism GraphPad version 6 and SPSS, version 22 software packages. All tests were two-tailed and exact $P$ values are reported with 3 decimal places (37).

\section{Results}

\section{Baseline characteristics}

Table 1 summarizes the characteristics of the two study groups. The PCOS and control groups had similar age, BMI, blood pressure, smoking and alcohol habits, physical activity, serum cholesterol, glucose and TSH levels. The only anthropometric variable that differed between groups was waist circumference, which was larger in PCOS (mean \pm S.D.: $91.0 \pm 12.3 \mathrm{~cm}$ ) than in controls $(82.4 \pm 10.9 \mathrm{~cm}, P=0.006)$. Among the PCOS patients, 28 were hyperandrogenic (of whom 26 had all three diagnostic criteria and 2 had hyperandrogenism with polycystic ovarian morphology but not menstrual irregularity) and 17 were normoandrogenic. 
Table 1 Characteristics of the study participants.

\begin{tabular}{l}
\hline \\
\hline Age (years) \\
Weight $(\mathrm{kg})$ \\
Height $(\mathrm{cm})$ \\
Body mass index $\left(\mathrm{kg} / \mathrm{m}^{2}\right)$ \\
Waist circumference $(\mathrm{cm})$ \\
Blood pressure (mmHg) \\
Systolic \\
Diastolic \\
Smoking $(n, \%)$ \\
Alcohol drinking ( $n, \%)$ \\
Physical activity duration (min/week) \\
Physical activity classification $(n, \%)$ \\
Very active \\
Active Sedentary \\
Current hypocaloric diet $(n, \%)$ \\
Serum cholesterol $(\mathrm{mg} / \mathrm{dL})$ \\
Total \\
HDL-c \\
Fasting Glucose $(\mathrm{mg} / \mathrm{dL})$ \\
Serum TSH ( $\mu$ IU/mL)
\end{tabular}

\begin{tabular}{c}
\hline $\operatorname{PCOS}(n=45)$ \\
\hline $30.6 \pm 5.5$ \\
$73.4 \pm 13.1$ \\
$161.1 \pm 6.2$ \\
$28.2 \pm 4.6$ \\
$91.0 \pm 12.3$
\end{tabular}

$110(110-120)$
$70(70-80)$
$6(13 \%)$
$10(22 \%)$
$400(180-635)$
$8(18 \%)$
$27(60 \%)$
$10(22 \%)$
$7(17 \%)$

$188.4 \pm 38.7$

$47.5 \pm 12.4$

$88.3+7.1$

$2.54 \pm 2.45$

\begin{tabular}{c}
\hline Control $(n=25)$ \\
\hline $33.2 \pm 6.7$ \\
$69.2 \pm 12.1$ \\
$160.4 \pm 6.3$ \\
$26.9 \pm 4.6$ \\
$82.4 \pm 10.9$ \\
$110(100-120)$ \\
$75(68-80)$ \\
$0(0 \%)$ \\
$3(12 \%)$ \\
$420(275-930)$ \\
$4(16 \%)$ \\
$15(60 \%)$ \\
$6(24 \%)$ \\
$4(16 \%)$
\end{tabular}

$184.3 \pm 16.2$

$54.1 \pm 11.4$

$92.4 \pm 7.8$

$2.95 \pm 0.79$

\begin{tabular}{c}
\hline Mean difference $(95 \% \mathrm{CI})$ \\
\hline$-2.6(-5.6$ to 0.3$)$ \\
$4.2(-2.2$ to 10.5$)$ \\
$0.8(-2.3$ to 3.9$)$ \\
$1.2(-1.1$ to 3.5$)$ \\
$8.5(2.6-14.5)$
\end{tabular}

\begin{tabular}{c}
\hline P value \\
\hline $0.083^{*}$ \\
$0.195^{*}$ \\
$0.613^{*}$ \\
$0.288^{*}$ \\
$0.006^{*}$
\end{tabular}

$0.557^{\dagger}$

$0.825^{\dagger)}$

$0.062^{\ddagger}$

$0.235^{\ddagger}$

$0.398^{\dagger}$

$0.822^{\S}$

$0.613^{\ddagger}$

$0.786^{*}$

$0.192^{*}$

$0.088^{*}$

$0.691^{*}$

Data are expressed as means \pm S.D. or medians (interquartile ranges). $P$ values refer to *Unpaired $t$ test, ${ }^{\dagger}$ Mann-Whitney test, ${ }^{\ddagger}$ Fisher's exact test or ${ }^{\S}$ chi-square test, as appropriate.

\section{BAT distribution and correlations}

Fig. 1 illustrates typical ${ }^{18} \mathrm{~F}$-FDG PET-CT images for detection of BAT in women with PCOS and healthy controls. Specific BAT activity was detected in all body regions evaluated and was present in $46 / 70$ (65.7\%) of the participants.
BAT activity was significantly reduced in women with PCOS (median SUV max $_{\text {ax }}$ 7.4, interquartile range: 0.9-15.4) compared to controls (median $\mathrm{SUV}_{\max }: 13.0$, interquartile range: 4.7-18.4, $P=0.047$, Mann-Whitney test, Fig. 2). When considering each anatomic region evaluated, the BAT activity level measured at region 3 (thorax) was
A

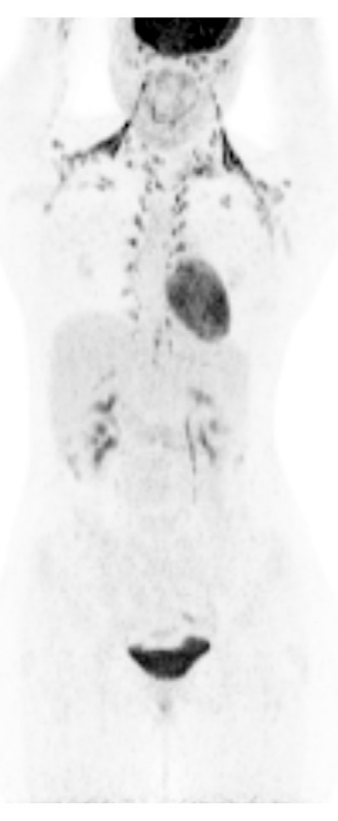

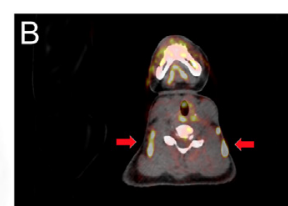
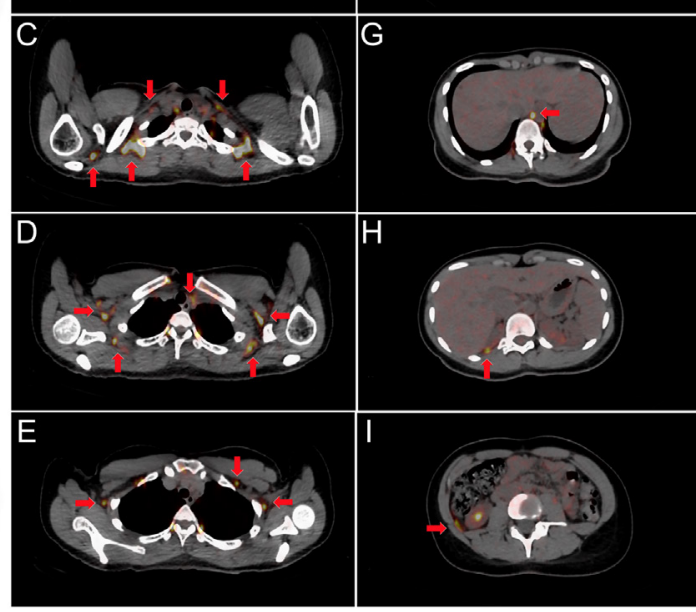

\section{Figure 1}

Typical localization of brown fat activity with ${ }^{18}$ F-FDG PET-CT. (A) Maximal intensity projection showing diffuse uptake of ${ }^{18} \mathrm{~F}$-FDG at different sites of brown fat. (B, $C, D, E, F, G, H$ and I) Representative axial slices throughout a patient's body showing sites of brown fat (red arrows). (B) Bilateral cervical regions; (C) bilateral cervico-thoracic transition; (D) bilateral cervico-thoracic transition, bilateral axillary regions; (E) bilateral axillary regions and anterior thoracic region; (F) mediastinal, bilateral axillary and bilateral intercostal regions; (G) thoracic-abdominal transition (close to esophagus); $(\mathrm{H})$ right perirenal space; (I) intraabdominal region. A full colour version of this figure is available at https://doi.org/10.1530/ EJE-19-0505. 
significantly reduced in women with PCOS (median $\mathrm{SUV}_{\max }$ : 1.0, interquartile range: 0.9-7.0) compared to controls $\left(\mathrm{SUV}_{\mathrm{max}}: 6.4\right.$, interquartile range: 1.0-10.8, $P=0.010$, Mann-Whitney test, Fig. 2). However, these differences were no longer significant when adjusted for waist circumference $(P=0.885$ and 0.205 for total BAT and region 3 , respectively).

Correlation analyses pooling PCOS women and controls showed that BAT activity was negatively correlated with BMI (Spearman's $r=-0.547, P=0.000)$ and waist circumference $(\mathrm{r}=-0.562, P=0.000)$. In addition, BAT was weakly and negatively correlated with plasma irisin levels ( $r=-0.266, P=0.026$, Fig. 2).

When considering only women with PCOS, BAT activity correlated inversely with BMI, waist circumference and LAP index in total and in most anatomical regions analyzed. Remarkably, in women with PCOS total BAT activity showed no correlation with age, serum testosterone, serum insulin or plasma irisin levels (Table 2). Secondary analysis did not show any significant difference in total BAT activity between hyperandrogenic
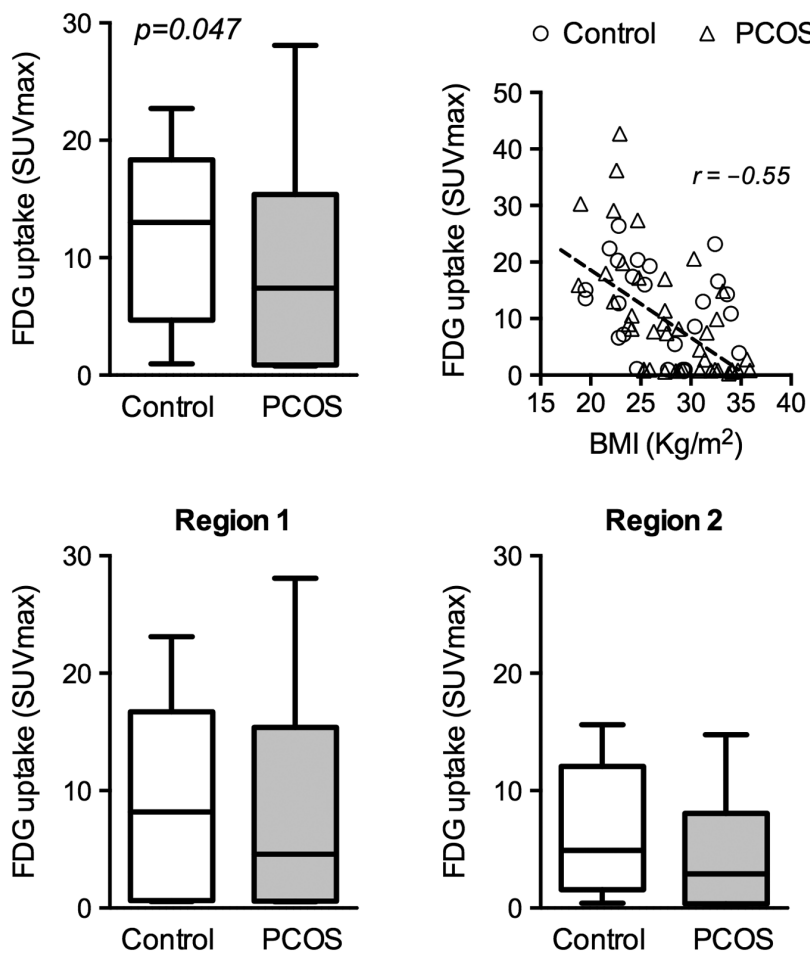

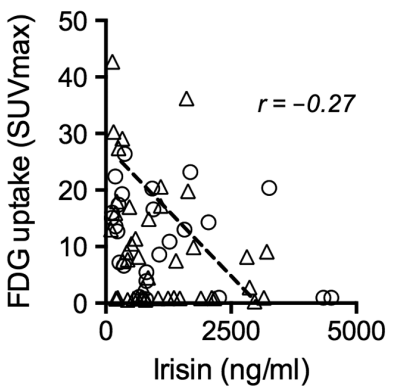

Region 3

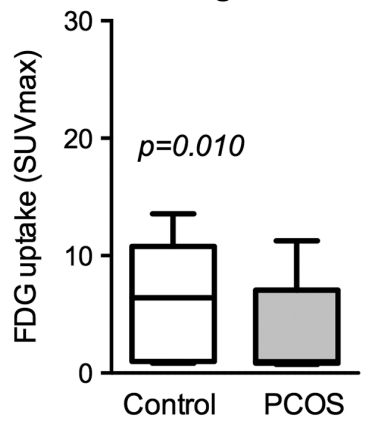

and normoandrogenic women with PCOS (adjusted $P=0.165)$.

\section{Plasma irisin levels}

Fig. 3 shows that plasma irisin levels were similar in women with PCOS and controls, but there was a positive correlation between irisin and BMI in the whole study sample $(r=0.581, P=0.000)$, as well as in each study group (PCOS, $r=0.542, P=0.000$; control, $r=0.662, P=0.000$ ).

\section{Discussion}

The main finding of our study was that patients with PCOS had a lower BAT activity than control patients. This finding was due to the decreased BAT activity in the thoracic region. We also found that BAT activity is inversely correlated with BMI and waist circumference but not with plasma irisin levels in PCOS women.

To the best of our knowledge, this is the first study that evaluated BAT activity and anatomical distribution

\section{Figure 2}

Quantification of brown adipose tissue activity by ${ }^{18}$ F-FDG uptake in control $(n=25)$ and PCOS $(n=45)$ groups. The boxplots represent the medians and quartiles, with error bars indicating the $10^{\text {th }}$ and $90^{\text {th }}$ percentiles. The scatter plots in the upper panel show negative correlations between total BAT and BMI, waist circumference and plasma irisin. The lower plots compare BAT activity in the two groups at Region 1 (cervico-thoracic), Region 2 (axillary), Region 3 (thoracic), and Region 4 (abdominal). $P$ values refer to Mann-Whitney test. 
Table 2 Linear correlation between brown adipose tissue activity at different body regions and some anthropometric and metabolic variables in PCOS women $(n=45)$.

\begin{tabular}{|c|c|c|c|}
\hline & Total & Region 1 cervico-thoracic & Region 2 axillary \\
\hline Age & $-0.241(0.111)$ & $-0.134(0.380)$ & $-0.148(0.331)$ \\
\hline Body mass index & $-0.630(0.000)$ & $-0.602(0.000)$ & $-0.675(0.000)$ \\
\hline Waist circumference & $-0.592(0.000)$ & $-0.503(0.001)$ & $-0.628(0.000)$ \\
\hline LAP index & $-0.392(0.022)$ & $-0.440(0.009)$ & $-0.549(0.001)$ \\
\hline Serum total testosterone & $0.162(0.383)$ & $0.151(0.419)$ & $0.112(0.549)$ \\
\hline Serum insulin & $-0.109(0.666)$ & $-0.083(0.745)$ & $-0.265(0.289)$ \\
\hline Plasma irisin & $-0.250(0.098)$ & $-0.263(0.081)$ & $-0.288(0.055)$ \\
\hline
\end{tabular}

\begin{tabular}{l}
\hline Region 3 thoracic \\
\hline$-0.136(0.374)$ \\
$-0.534(0.000)$ \\
$-0.590(0.000)$ \\
$-0.307(0.077)$ \\
$0.303(0.097)$ \\
$-0.176(0.486)$ \\
$-0.257(0.089)$ \\
\hline
\end{tabular}

\begin{tabular}{c}
\hline Region 4 abdominal \\
\hline$-0.007(0.961)$ \\
$-0.498(0.001)$ \\
$-0.437(0.003)$ \\
$-0.359(0.037)$ \\
$0.149(0.423)$ \\
$-0.075(0.767)$ \\
$-0.251(0.097)$
\end{tabular}

Data are presented as Spearman's rank correlation coefficient and $P$ values are shown in parentheses.

in women with PCOS using ${ }^{18}$ F-FDG PET-CT. However, a recent study detected lower skin temperature at supraclavicular region in women with PCOS, which also suggests lower BAT activity compared to controls (38). Based on studies involving other metabolic diseases, we hypothesized that BAT activity might be lower in women with PCOS. In patients with cardiovascular disease (39) or pheochromocytoma (40) the presence of BAT has been negatively associated with central obesity and metabolic dysfunction. Other studies have shown that BAT activity is negatively correlated with visceral fat area (8) and proportion of fat body mass (4) in healthy volunteers.

Here we have expanded this observation to women with PCOS, whose BAT activity was decreased in comparison to controls and was inversely correlated with waist circumference, a surrogate for central adiposity. By using axial magnetic resonance imaging at selected anatomical sites, Barber et al. found the same amount of visceral fat in women with and without PCOS (41). However, their groups were matched for fat mass and had equal mean waist circumference, whereas ours were balanced by age and BMI but differed in waist circumference, which was larger in the PCOS group. Thus, it is uncertain whether the lower BAT activity is directly related to PCOS or whether it is associated with visceral adiposity, since the difference between groups does not hold when the results are adjusted for waist circumference. Furthermore,
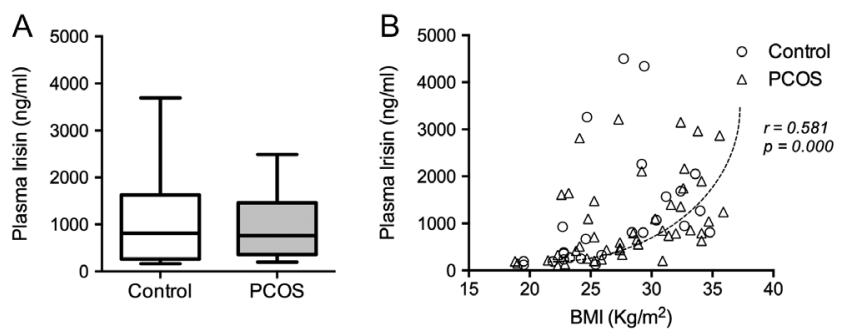

Figure 3

Plasma irisin levels in control $(n=25)$ and PCOS $(n=45)$ groups and their correlation with BMI. the lack of correlation between BAT activity and plasma irisin levels in the PCOS group suggests that the lower BAT activity is not explainable by low irisin levels.

The lower activity of brown fat identified in PCOS patients when compared to healthy women was due to differences in the thoracic region. We hypothesize that this might be due to some topographic variation of hormonal mechanisms affecting human BAT metabolism, which however remains to be investigated. Furthermore, there was an inverse correlation between BAT and LAP index, an insulin resistance and cardiovascular risk marker (31). Surprisingly there was no relationship between serum testosterone and BAT activity in any of the studied regions, suggesting that the decrease in brown fat is related to a metabolic issue independent of changes in the hypothalamic-pituitaryovary axis, and that the reproductive changes of PCOS may not affect the regulation of the browning pathway of white adipose tissue. Accordingly, supraclavicular skin temperature, which indirectly measures BAT activity, was found weakly associated with testosterone levels in women with PCOS (38). Nevertheless, a link between androgens and BAT activity cannot be ruled out since we measured serum testosterone with an immunoassay, which is not as accurate as the gold standard chromatography-mass spectrometry, and we did not assess androgen precursors such as androstenedione and dehydroepiandrosterone sulfate.

Studies suggested that, compared to white fat, BAT depots are less susceptible to developing local inflammation in response to obesity. However, strong obesogenic insults ultimately induce a local pro-inflammatory environment in brown fat. In mice, this condition directly alters the thermogenic activity of BAT by impairing its energy expenditure mechanism and reducing glucose uptake for use as a fuel substrate (42). This mechanism might justify the lower BAT activity in PCOS patients as the syndrome is often associated with chronic low-grade inflammation (43).

There is growing interest in exploring circulating irisin levels in PCOS patients, as many studies demonstrated 
that irisin can be involved with PCOS pathophysiology (22). In the current study, PCOS women had similar plasma irisin concentrations in comparison to the control group. In the literature, the characterization of irisin in PCOS is quite controversial since normal (44), increased $(18,21,23)$ and decreased (16) irisin concentrations have been described in these patients. This inconsistency may be partly due to differences in the assay methods, but it may also be due to the interference of confounding variables associated with PCOS. Recently, two metaanalyses have summarized irisin levels in women with PCOS. The first meta-analysis included eight studies involving 1918 PCOS patients and 528 controls. The pooled data indicated that the levels of irisin were higher in patients with PCOS and were unrelated to insulin resistance (45). The other meta-analysis included eight studies with 918 PCOS patients and 528 healthy controls and demonstrated that, when adjusted for BMI, circulating irisin in PCOS patients seems comparable to healthy controls (22).

One strength of the present study is that the PCOS and control groups were matched by age and BMI and were similar except by abdominal circumference, which is often increased in PCOS $(31,46)$. In addition, PET-CT examinations were performed by the same professionals unaware of the participant's group, while plasma irisin assays were performed blindly and by a widely validated technique. Our study also managed to recruit the number of participants calculated to achieve statistical power in the comparisons between PCOS and control groups. One limitation of this study is the cross-sectional design that does not allow us to define whether the lower BAT activity was present before the onset of PCOS symptoms. Another limitation is that androgen levels were assessed only by total testosterone immunoassay.

In conclusion, BAT activity was reduced in women with PCOS possibly due to increased central adiposity. In PCOS women, BAT activity did not correlate with plasma irisin levels.

\section{Declaration of interest}

The authors declare that there is no conflict of interest that could be perceived as prejudicing the impartiality of this study.

\section{Funding}

Research supported by Fundação de Amparo à Pesquisa do Estado de Minas Gerais (FAPEMIG, grant \# APQ-03798-16) and Conselho Nacional de Desenvolvimento Científico e Tecnológico (CNPq) through the National Institute of Hormones and Women's Health and the National Institute of Molecular Medicine. Publication was supported by Universidade Federal de Minas Gerais. K.B.G and F.M.R. receive research grants from CNPq. M.F.B. received a scholarship from CAPES.

\section{References}

1 Nicholls DG, Bernson VS \& Heaton GM. The identification of the component in the inner membrane of brown adipose tissue mitochondria responsible for regulating energy dissipation. Experientia. Supplementum 197832 89-93. (https://doi. org/10.1007/978-3-0348-5559-4_9)

2 Rothwell NJ \& Stock MJ. Effects of age on diet-induced thermogenesis and brown adipose tissue metabolism in the rat. International Journal of Obesity 19837 583-589.

3 Gesta S, Tseng YH \& Kahn CR. Developmental origin of fat: tracking obesity to its source. Cell 2007 131 242-256. (https://doi. org/10.1016/j.cell.2007.10.004)

4 van Marken Lichtenbelt WD, Vanhommerig JW, Smulders NM, Drossaerts JM, Kemerink GJ, Bouvy ND, Schrauwen P \& Teule GJ. Cold-activated brown adipose tissue in healthy men. New England Journal of Medicine 2009360 1500-1508. (https://doi.org/10.1056/ NEJMoa0808718)

5 Jung RT, Leslie P, Nicholls DG, Cunningham S \& Isles TE. Energy expenditure in normal and diabetic man: the role of brown adipose tissue. Health Bulletin 198846 55-62.

6 Cypess AM, Lehman S, Williams G, Tal I, Rodman D, Goldfine AB, Kuo FC, Palmer EL, Tseng YH, Doria A et al. Identification and importance of brown adipose tissue in adult humans. New England Journal of Medicine 2009360 1509-1517. (https://doi.org/10.1056/ NEJMoa0810780)

7 Virtanen KA, Lidell ME, Orava J, Heglind M, Westergren R, Niemi T, Taittonen M, Laine J, Savisto NJ, Enerback S et al. Functional brown adipose tissue in healthy adults. New England Journal of Medicine 2009360 1518-1525. (https://doi.org/10.1056/NEJMoa0808949)

8 Saito M, Okamatsu-Ogura Y, Matsushita M, Watanabe K, Yoneshiro T, Nio-Kobayashi J, Iwanaga T, Miyagawa M, Kameya T, Nakada K et al. High incidence of metabolically active brown adipose tissue in healthy adult humans: effects of cold exposure and adiposity. Diabetes 200958 1526-1531. (https://doi.org/10.2337/ db09-0530)

9 Nedergaard J, Bengtsson T \& Cannon B. Unexpected evidence for active brown adipose tissue in adult humans. American Journal of Physiology: Endocrinology and Metabolism 2007293 E444-E452. (https://doi.org/10.1152/ajpendo.00691.2006)

10 Hany TF, Gharehpapagh E, Kamel EM, Buck A, Himms-Hagen J \& von Schulthess GK. Brown adipose tissue: a factor to consider in symmetrical tracer uptake in the neck and upper chest region. European Journal of Nuclear Medicine and Molecular Imaging 200229 1393-1398. (https://doi.org/10.1007/s00259-002-0902-6)

11 Rosenfield RL \& Ehrmann DA. The pathogenesis of polycystic ovary syndrome (PCOS): the hypothesis of PCOS as functional ovarian hyperandrogenism revisited. Endocrine Reviews 201637 467-520. (https://doi.org/10.1210/er.2015-1104)

12 Villa J \& Pratley RE. Adipose tissue dysfunction in polycystic ovary syndrome. Current Diabetes Reports 201111 179-184. (https://doi. org/10.1007/s11892-011-0189-8)

13 Yuan X, Hu T, Zhao H, Huang Y, Ye R, Lin J, Zhang C, Zhang H, Wei $\mathrm{G}$, Zhou $\mathrm{H}$ et al. Brown adipose tissue transplantation ameliorates polycystic ovary syndrome. PNAS 2016113 2708-2713. (https://doi.org/10.1073/pnas.1523236113)

14 Pedersen BK \& Febbraio MA. Muscles, exercise and obesity: skeletal muscle as a secretory organ. Nature Reviews Endocrinology 20128 457-465. (https://doi.org/10.1038/nrendo.2012.49)

15 Bostrom P, Wu J, Jedrychowski MP, Korde A, Ye L, Lo JC, Rasbach KA, Bostrom EA, Choi JH, Long JZ et al. A PGC1-alpha-dependent myokine that drives brown-fat-like development of white fat and thermogenesis. Nature $2012 \mathbf{4 8 1} 463-468$. (https://doi.org/10.1038/ nature10777)

16 Abali R, Temel Yuksel I, Yuksel MA, Bulut B, Imamoglu M, Emirdar V, Unal F, Guzel S \& Celik C. Implications of circulating irisin and Fabp4 levels in patients with polycystic ovary syndrome. Journal of 
Obstetrics and Gynaecology 201636 897-901. (https://doi.org/10.3109 /01443615.2016.1174200)

17 Polyzos SA, Anastasilakis AD, Efstathiadou ZA, Makras P, Perakakis N, Kountouras J \& Mantzoros CS. Irisin in metabolic diseases. Endocrine 201859 260-274. (https://doi.org/10.1007/s12020-017-1476-1)

18 Li M, Yang M, Zhou X, Fang X, Hu W, Zhu W, Wang C, Liu D, Li S, Liu $\mathrm{H}$ et al. Elevated circulating levels of irisin and the effect of metformin treatment in women with polycystic ovary syndrome. Journal of Clinical Endocrinology and Metabolism 2015100 1485-1493. (https://doi.org/10.1210/jc.2014-2544)

19 Huh JY, Panagiotou G, Mougios V, Brinkoetter M, Vamvini MT, Schneider BE \& Mantzoros CS. FNDC5 and irisin in humans: I. Predictors of circulating concentrations in serum and plasma and II. mRNA expression and circulating concentrations in response to weight loss and exercise. Metabolism: Clinical and Experimental 2012 61 1725-1738. (https://doi.org/10.1016/j.metabol.2012.09.002)

20 Moreno-Navarrete JM, Ortega F, Serrano M, Guerra E, Pardo G, Tinahones F, Ricart W \& Fernandez-Real JM. Irisin is expressed and produced by human muscle and adipose tissue in association with obesity and insulin resistance. Journal of Clinical Endocrinology and Metabolism 201398 E769-E778. (https://doi.org/10.1210/jc.2012-2749)

21 Zhang L, Fang X, Li L, Liu R, Zhang C, Liu H, Tan M \& Yang G. The association between circulating irisin levels and different phenotypes of polycystic ovary syndrome. Journal of Endocrinological Investigation 201841 1401-1407. (https://doi.org/10.1007/s40618-018-0902-4)

22 Cai X, Qiu S, Li L, Zugel M, Steinacker JM \& Schumann U. Circulating irisin in patients with polycystic ovary syndrome: a metaanalysis. Reproductive Biomedicine Online 201836 172-180. (https:// doi.org/10.1016/j.rbmo.2017.10.114)

23 Chang CL, Huang SY, Soong YK, Cheng PJ, Wang CJ \& Liang IT. Circulating irisin and glucose-dependent insulinotropic peptide are associated with the development of polycystic ovary syndrome. Journal of Clinical Endocrinology and Metabolism 201499 E2539E2548. (https://doi.org/10.1210/jc.2014-1180)

24 Costanza MC. Matching. Preventive Medicine 199524 425-433. (https://doi.org/10.1006/pmed.1995.1069)

25 Rotterdam EA-SPCWG. Revised 2003 consensus on diagnostic criteria and long-term health risks related to polycystic ovary syndrome. Fertility and Sterility 200481 19-25.

26 Craig CL, Marshall AL, Sjostrom M, Bauman AE, Booth ML, Ainsworth BE, Pratt M, Ekelund U, Yngve A, Sallis JF et al. International physical activity questionnaire: 12 -country reliability and validity. Medicine and Science in Sports and Exercise 200335 1381-1395. (https://doi.org/10.1249/01.MSS.0000078924.61453.FB)

27 Macfarlane DJ, Lee CC, Ho EY, Chan KL \& Chan D. Convergent validity of six methods to assess physical activity in daily life. Journal of Applied Physiology 2006101 1328-1334. (https://doi.org/10.1152/ japplphysiol.00336.2006)

28 Colpani V, Oppermann K \& Spritzer PM. Causes of death and associated risk factors among climacteric women from Southern Brazil: a population based-study. BMC Public Health 201414194 (https://doi.org/10.1186/1471-2458-14-194)

29 Bastos CA, Oppermann K, Fuchs SC, Donato GB \& Spritzer PM. Determinants of ovarian volume in pre-, menopausal transition, and post-menopausal women: a population-based study. Maturitas 2006 53 405-412. (https://doi.org/10.1016/j.maturitas.2005.07.002)

30 Lauria PB, Del Puerto HL, Reis AM, Candido AL \& Reis FM. Low plasma atrial natriuretic peptide: a new piece in the puzzle of polycystic ovary syndrome. Journal of Clinical Endocrinology and Metabolism 201398 4882-4889. (https://doi.org/10.1210/jc.2013-2141)

31 Wiltgen D, Benedetto IG, Mastella LS \& Spritzer PM. Lipid accumulation product index: a reliable marker of cardiovascular risk in polycystic ovary syndrome. Human Reproduction 200924 1726-1731. (https://doi.org/10.1093/humrep/dep072)

32 Martinez-Tellez B, Sanchez-Delgado G, Garcia-Rivero Y, Alcantara JMA, Martinez-Avila WD, Munoz-Hernandez MV, Olza J, Boon MR, Rensen PCN, Llamas-Elvira JM et al. A new personalized cooling protocol to activate brown adipose tissue in young adults. Frontiers in Physiology 20178863.

33 Engineers ASoHRaA-C. 2009 ASHRAE Handbook - Fundamentals. ASHRAE, 2009.

34 ToolBox E. Clo - Clothing and Thermal Insulation, 2004.

35 Sugawara Y, Zasadny KR, Neuhoff AW \& Wahl RL. Reevaluation of the standardized uptake value for FDG: variations with body weight and methods for correction. Radiology 1999213 521-525. (https:// doi.org/10.1148/radiology.213.2.r99nv37521)

36 Pourhoseingholi MA, Baghestani AR \& Vahedi M. How to control confounding effects by statistical analysis. Gastroenterology and Hepatology from Bed to Bench 20125 79-83.

37 Dekkers OM. Why not to (over)emphasize statistical significance. European Journal of Endocrinology 2019181 E1-E2. (https://doi. org/10.1530/EJE-19-0531)

38 Shorakae S, Jona E, de Courten B, Lambert GW, Lambert EA, Phillips SE, Clarke IJ, Teede HJ \& Henry BA. Brown adipose tissue thermogenesis in polycystic ovary syndrome. Clinical Endocrinology 201990 425-432. (https://doi.org/10.1111/cen.13913)

39 Franssens BT, Hoogduin H, Leiner T, van der Graaf Y \& Visseren FLJ. Relation between brown adipose tissue and measures of obesity and metabolic dysfunction in patients with cardiovascular disease. Journal of Magnetic Resonance Imaging 201746 497-504. (https://doi. org/10.1002/jmri.25594)

40 Wang Q, Zhang M, Ning G, Gu W, Su T, Xu M, Li B \& Wang W. Brown adipose tissue in humans is activated by elevated plasma catecholamines levels and is inversely related to central obesity. PLOS ONE 20116 e21006. (https://doi.org/10.1371/journal. pone.0021006)

41 Barber TM, Golding SJ, Alvey C, Wass JA, Karpe F, Franks S \& McCarthy MI. Global adiposity rather than abnormal regional fat distribution characterizes women with polycystic ovary syndrome. Journal of Clinical Endocrinology and Metabolism 200893 999-1004. (https://doi.org/10.1210/jc.2007-2117)

42 Villarroya F, Cereijo R, Gavalda-Navarro A, Villarroya J \& Giralt M. Inflammation of brown/beige adipose tissues in obesity and metabolic disease. Journal of Internal Medicine 2018284 492-504. (https://doi.org/10.1111/joim.12803)

43 Spritzer PM, Lecke SB, Satler F \& Morsch DM. Adipose tissue dysfunction, adipokines, and low-grade chronic inflammation in polycystic ovary syndrome. Reproduction 2015149 R219-R227. (https://doi.org/10.1530/REP-14-0435)

44 Pukajlo K, Laczmanski Ł, Kolackov K, Kuliczkowska-Plaksej J, Bolanowski M, Milewicz A \& Daroszewski J. Irisin plasma concentration in PCOS and healthy subjects is related to body fat content and android fat distribution. Gynecological Endocrinology 201531 907-911. (https://doi.org/10.3109/09513590. 2015.1065482)

45 Wang C, Zhang XY, Sun Y, Hou XG \& Chen L. Higher circulating irisin levels in patients with polycystic ovary syndrome: a metaanalysis. Gynecological Endocrinology 201834 290-293. (https://doi. org/10.1080/09513590.2017.1393065)

46 Couto Alves A, Valcarcel B, Makinen VP, Morin-Papunen L, Sebert S, Kangas AJ, Soininen P, Das S, De Iorio M, Coin L et al. Metabolic profiling of polycystic ovary syndrome reveals interactions with abdominal obesity. International Journal of Obesity 201741 13311340. (https://doi.org/10.1038/ijo.2017.126) 